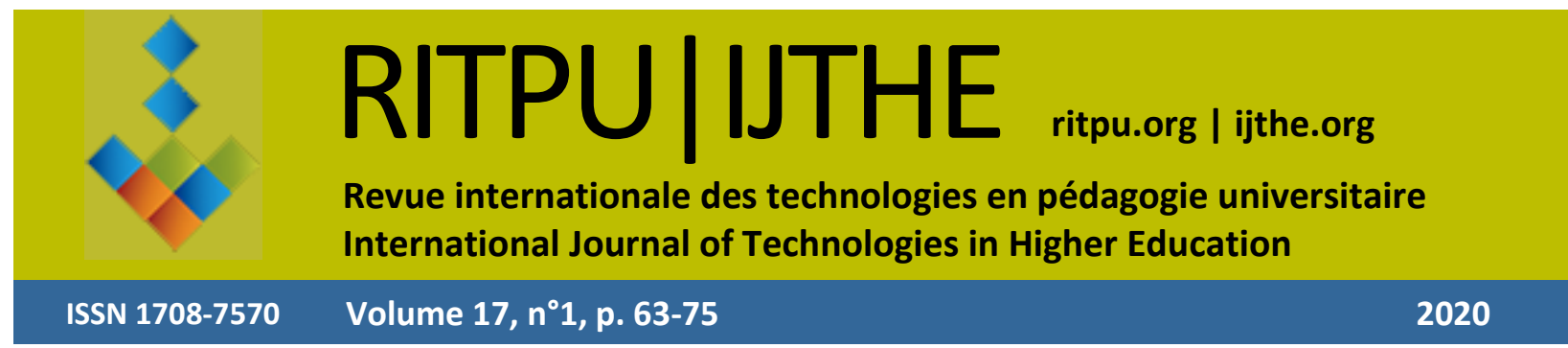

\section{Le développement professionnel au postsecondaire à l'ère du numérique}

\author{
Professional Development in Higher Education \\ in the Digital Age
}

https://doi.org/10.18162/ritpu-2020-v17n1-13
Normand ROY

normand.roy@umontreal.ca

Édith GRUSLIN

edith.gruslin@umontreal.ca

Bruno POELLHUBER

bruno.poellhuber@umontreal.ca

Université de Montréal

\title{
Résumé
}

Être enseignant à l'ère du numérique dépasse largement le contexte de la classe et s'inscrit dans une réalité de plus en plus complexe au postsecondaire (collégial et universitaire), notamment par une multiplication de l'offre de formation en ligne. Les enseignants du postsecondaire sont d'abord des spécialistes de contenu (Bolduc, 2010) et la formation initiale en éducation n'est pas obligatoire pour eux. Alors que le besoin de formation en éducation est partiellement comblé par une offre de formation créditée et non créditée par les établissements d'enseignement supérieur, nous observons actuellement une décentralisation et un éclatement de cette offre. S'inscrivant en cohérence avec le Plan d'action numérique et le Cadre de référence de la compétence numérique, le présent texte explicitera concrètement les liens entre l'état de la connaissance en développement professionnel et les potentialités du numérique.

\section{Mots-clés}

Développement professionnel, compétence numérique, collégial, universitaire

\section{Abstract}

Being a teacher in digital age goes well beyond the classroom and is part of a reality that is becoming increasingly complex at the post-secondary level (college and university), particularly with the multiplication of online training opportunities. Post-secondary teachers are primarily content specialists (Bolduc, 2010) and educational training is not mandatory for them. While the need for educational training is partially met by a supply of credited and non-credited training by higher education institutions, we are now seeing a decentralization and fragmentation of the offer. In line with the Digital Action Plan and the Digital Competency Framework, this text will explain the links between professional development expectancies and the potential of digital technology.

\section{Keywords}

Professional development, digital literacy, higher education 


\section{Contexte}

Le développement professionnel est une composante essentielle du métier d'enseignant et le contexte actuel de la COVID-19 met en exergue sa grande nécessité. En 2014, le Conseil supérieur de l'éducation (CSE) s'est penché sur la question, en proposant un rapport intitulé Le développement professionnel, un enrichissement pour toute la profession enseignante. Au fil des 232 pages, les auteurs explorent toute la complexité du champ, en insistant d'abord sur les définitions du concept, en proposant ensuite l'état des lieux et des données empiriques sur le sujet, pour finalement conclure sur de grandes orientations pour les années à venir. Or, ce rapport ne s'intéresse pas particulièrement au cas spécifique du postsecondaire, qui s'inscrit, selon nous, dans une réalité différente des autres ordres d'enseignement.

Les enseignants et enseignantes des niveaux collégial et universitaire sont d'abord et avant tout des spécialistes de contenu (Bolduc, 2010), avec des formations très variables en pédagogie (St-Pierre et Lison, 2009). Dès 2000, le CSE reconnait l'importance de soutenir le développement professionnel des membres du personnel enseignant au postsecondaire, sur le plan tant financier que pédagogique. Les collèges et les universités doivent mutuellement se soutenir pour proposer une offre de formation concertée. Dans le rapport, les auteurs abordent les technologies comme un des objets du développement professionnel, mais très peu comme un moyen pour le réaliser (CSE, 2014). Le présent texte s'intéresse plutôt à ce dernier contexte, soit la place du numérique comme vecteur de transformation des pratiques professionnelles et non comme objet du développement professionnel, ce que de nombreux textes ont déjà étudié (Karsenti et Grégoire, 2015; Raby et al., 2011).

Sans l'ombre d'un doute, le numérique, du fait de sa nature évolutive, modifie les possibilités de développement professionnel des enseignants et implique également une mise à jour continuelle. Ainsi, le numérique est autant le moyen utilisé pour mettre en place le changement (utiliser le numérique pour développer les compétences professionnelles) qu'il en est la cause (développer sa compétence numérique). Dans ce contexte, le document d'orientation La formation à l'enseignement du ministère de l'Éducation du Québec (Martinet et al., 2001) soutient que l'enseignant doit «[s]'engager dans une démarche individuelle et collective de développement professionnel » (compétence 11, p. 156). Quant au nouveau Cadre de référence de la compétence numérique (Ministère de l'Éducation et de l'Enseignement supérieur [MEES], 2019), il suggère que le numérique permet «d'adopter une perspective de développement personnel et professionnel avec le numérique dans une posture d'autonomisation » (dimension 9, p. 21).

De nombreux réseaux s'intéressent particulièrement aux usages du numérique, et ce, dans tous les milieux scolaires. Par exemple, le Réseau des répondantes et répondants TIC (Réseau REPTIC, s.d.) est « une communauté de pratique qui regroupe, anime et appuie les conseillères et conseillers pédagogiques responsables de l'intégration pédagogique des technologies dans les cégeps et collèges publics et privés du Québec ». Ceux-ci sont au cœur de multiples stratégies pour favoriser le développement des compétences professionnelles des enseignants. Leurs rencontres régulières permettent aux acteurs du milieu de partager et d'échanger par le biais de conférences portant sur différentes pratiques pédagogiques. Cette communauté de pratique fonctionne, entre ses rencontres, avec une liste de distribution très active, une mise à jour continue du site Web et des groupes de travail sur des sujets particuliers.

Toutefois, les technologies comme moyen de développement professionnel gagnent à être davantage explorées. La situation découlant de la crise sanitaire causée par la COVID-19 nous permet d'assister à une recrudescence phénoménale des formations en ligne et des 
accompagnements virtuels. Toutefois, le CSE (2014, p. 86) souligne qu'il faut s'interroger sur l'efficacité des enseignants à user des ressources existantes pour favoriser leur développement professionnel. Deschênes (2014) suggère d'ailleurs que le Web social serait un levier de développement professionnel pertinent. C'est également l'un des constats de Hamel et al. (2013), pour qui le soutien technologique (notamment la conférence Web et le forum) a été un levier important de transformation des pratiques dans l'initiative École éloignée en réseau. L'objectif de ce texte est d'examiner les usages du numérique dans le contexte d'un développement professionnel efficace des enseignants du postsecondaire. Nous décrirons d'abord brièvement ce que nous entendons par développement professionnel pour ensuite proposer une liste de moyens favorisant ce dernier en cohérence avec les caractéristiques des meilleures pratiques.

\section{Le développement professionnel : un terme polysémique}

Nous nous inscrivons dans la continuité des définitions proposées par le CSE (2014) et par Uwamariya et Mukamurera (2005), à savoir qu'il s'agit d'un processus de changement et de transformation, afin d'amener les enseignants à mieux maitriser leur travail et à s'y sentir à l'aise. Ce processus s'inscrit dans une dynamique individuelle et en collaboration avec les autres professionnels, pour trouver des solutions aux problèmes rencontrés. $\mathrm{Au}$ carrefour de nombreuses postures épistémologiques, le développement professionnel s'appuie sur deux perspectives : développementale et professionnalisante. Dans la perspective développementale, l'enseignant est vu comme quelqu'un qui développe sa compétence à travers une "succession de stades comportant des caractéristiques ou des changements qui leur sont propres » (Uwamariya et Mukamurera, 2005, p. 148). Dans la perspective professionnalisante, on insiste davantage sur la pratique réflexive et le caractère systémique et continu du développement professionnel, où la « construction d'une professionnalité qui met en valeur l'acquisition d'un ensemble de savoirs pertinents à l'enseignement » (p. 148) est au cœur du développement de l'enseignant.

Le développement professionnel peut être guidé par des objectifs de différentes natures en lien avec les activités d'apprentissage, qui couvrent un large éventail de possibilités : la formation des connaissances, l'encadrement, l'intégration, la planification, etc. (CSE, 2014, tableau 1, p. 19). Nonobstant les vastes perspectives pour le développement des compétences professionnelles, les stratégies employées quant à elles sont plus limitées et s'organisent sous différentes catégories. Il est possible de situer les stratégies de développement professionnel sur un continuum allant des stratégies transmissives à celles qui sont transformatrices (Kennedy, 2005, 2014). Entre les deux pôles de ce continuum se situent des stratégies dont l'objectif est "malléable », c'est-à-dire qu'elles peuvent être utilisées avec une visée soit transmissive, soit transformatrice (Kennedy, 2014).

\section{Le développement professionnel par le biais du numérique}

Comme nous l'avons mentionné précédemment, l'objectif principal de ce texte est d'expliciter les usages possibles du numérique pour favoriser le développement professionnel des enseignants au postsecondaire en fonction des différentes visées possibles. Pour atteindre notre objectif, nous présenterons différents moyens particulièrement intéressants et efficaces : les massive open online courses ( MOOC »), les visioconférences Web, les réseaux sociaux (Twitter et Facebook) et les projets intégrant une variété de moyens (communauté d'apprentissage, communauté professionnelle, etc.).

Cinq caractéristiques du développement professionnel semblent faire consensus et être associées à l'apprentissage et au développement d'habiletés des enseignants dans le contexte de développement professionnel: 1) la durée, 2) la thématique précise (content focus), 3) la 
cohérence, 4) l'apprentissage actif et 5) la participation collective (Desimone, 2009). La durée concerne tant le nombre d'heures que la période sur laquelle elles sont réparties. Bien que les résultats de la recherche associant la durée prolongée à un développement professionnel efficace soient parfois faibles (Stes et al, 2010), Desimone (2009) indique que les changements prennent du temps à s'installer et que des activités d'une durée de vingt heures ou plus réparties sur au moins un trimestre semblent favorables au développement professionnel. Le contenu pertinent et la cohérence perçue avec les connaissances, croyances et buts personnels ainsi qu'avec le contexte et les politiques institutionnelles et gouvernementales semblent aussi favoriser le développement professionnel (Garet et al., 2001; Penuel et al., 2007; Wei et al., 2009). L'apprentissage actif et la collaboration émergeant de la participation collective, dont les bénéfices ont été largement démontrés auprès des étudiants (Beichner et al., 2007; Freeman et al., 2014; Prince, 2004), semblent aussi avoir des impacts positifs sur l'apprentissage des enseignants lors du développement professionnel (Garet et al., 2001; Gast et al., 2017; Patton et al, 2013).

Le développement professionnel avec le numérique s'inscrit en directe cohérence avec la dimension 9 du Cadre de référence de la compétence numérique (MEES, 2019). Nous nous intéresserons plus particulièrement au premier élément, soit « acquérir, maintenir ou développer des compétences pour sa vie professionnelle actuelle ou future à l'aide du numérique ». Nous proposons de porter un regard critique sur différents moyens technologiques pouvant favoriser le développement des compétences professionnelles en fonction des cinq caractéristiques décrites ci-dessus.

\section{Les types de stratégies}

\section{Les stratégies transmissives}

Les stratégies transmissives regroupent des stratégies où l'information est transmise aux enseignants par un « expert » qui est souvent responsable de l'établissement des objectifs et du déroulement, seul ou avec les planificateurs de la formation (Guskey, 2000; Kennedy, 2005). Elles ont l'avantage de faciliter la dissémination d'idées chez un grand nombre d'individus, et ce, à faible coût (Guskey, 2000). Elles permettent ainsi à plusieurs enseignants de partager un vocabulaire commun ou de faire connaitre de nouveaux contenus ou méthodes disciplinaires ciblés. Les exemples de ce type de stratégies abondent en enseignement postsecondaire : ateliers, journées pédagogiques, communications dans des colloques professionnels ou scientifiques. Par contre, celles-ci offrent au participant peu de choix (Guskey, 2000) et un faible soutien à l'autonomie (Fraser et al., 2007). En fait, l'apprenant se retrouve dans un contexte fortement scénarisé, avec une pédagogie centrée sur l'enseignant. Il n'a que très peu de liberté et doit répondre aux exigences prévues au dispositif. De plus, les stratégies transmissives tendent fréquemment à maintenir le participant dans une position passive et à encourager un apprentissage individuel (Olmstead et Turpen, 2016). Enfin, les activités de formation de courte durée sont souvent critiquées et considérées comme peu efficaces pour mener à des changements de pratiques (Garet et al., 2001; Stes et al., 2010). Pour ces auteurs, elles ne permettent pas une réelle évolution dans le milieu de pratique ni une transformation à long terme des gestes professionnels.

\section{Les visioconférences Web et les vidéos pédagogiques}

Parmi les stratégies à visées transmissives faisant appel au numérique se trouvent les visioconférences et les vidéos pédagogiques diffusées sur le Web. Avec la venue de l'Internet à haute vitesse ont émergé la possibilité de transférer une grande quantité de données ainsi que des 
moyens de communication interactifs qui permettent la perception des signes non verbaux. Ces dernières années, les outils de visioconférence Web se sont multipliés et leur performance s'est grandement améliorée. Cela a ouvert la porte à une offre de formation en ligne de type webinaire, où les experts peuvent rejoindre un large public. Le REPTIC et l'APOP offrent notamment des webinaires pour présenter des ressources et expliciter les possibilités pédagogiques (Réseau REPTIC, s.d.-b). Le Centre de recherche interuniversitaire sur la formation et la profession enseignante (CRIFPE) propose la majorité de ses activités de formation en mode présentiel et en ligne, permettant ainsi le partage d'expertise des chercheurs du réseau. Les conférences deviennent ensuite accessibles en ligne, les enregistrements pouvant être rendus disponibles de façon asynchrone. La visioconférence Web occupe une place dans les mesures alternatives à l'enseignement en présentiel mises en place par plusieurs établissements d'enseignement postsecondaire dans le contexte de la crise de la COVID-19. Les formes des conférences Web peuvent être assez diversifiées: conférences Web, vidéos de pratiques exemplaires, témoignages, etc. Pour le développement professionnel, l'accessibilité aux experts de façon synchrone et asynchrone représente une grande richesse de formation, tout en pouvant s'inscrire en cohérence avec les besoins actuels des enseignants. Cependant, cette stratégie habituellement transmissive est souvent de courte durée et laisse une place limitée à l'apprentissage actif et collaboratif du participant.

\section{Les stratégies malléables}

Les stratégies dites malléables peuvent viser des objectifs transmissifs ou transformateurs, selon la philosophie qui les sous-tend, la forme qu'elles prennent et les relations qui s'y instaurent (Fraser et al., 2007; Kennedy, 2014). Ainsi, une même stratégie pourrait, par exemple, soutenir l'autonomie et la créativité ou, à l'inverse, prôner le statu quo. La stratégie des communautés d'apprentissage prend en compte l'aspect social de l'apprentissage et reconnait que les interactions dans la communauté sont elles-mêmes source d'apprentissage (Fraser et al., 2007; Kennedy, 2014). Les communautés d'apprentissage peuvent contribuer à disséminer de l'information, à renforcer des croyances communes, voire à modifier le pratiques des participants. Les membres peuvent y participer plus ou moins activement. Elles sont ainsi considérées comme des stratégies à visées malléables puisque l'orientation de la communauté et la participation individuelle peuvent avoir des visées et des retombées différentes (Kennedy, 2005). Elles suggèrent une implication sur une longue période de temps, ce qui peut être perçu comme un inconvénient par des participants ou même par des établissements d'enseignement.

\section{Les MOOC ${ }^{1}$}

Les cours en ligne ouverts à tous, accessibles en ligne gratuitement, peuvent s'inscrire autant dans les approches transmissives que transformatrices. Ils sont le plus souvent conçus d'une manière très transmissive (xMOOC), mais ont une durée considérable, soit de deux à quatre mois. Certains d'entre eux (cMOOC) proposent cependant différentes modalités de collaboration, en utilisant des réseaux sociaux ou des forums de discussion. Les MOOC rejoignent des millions d'apprenants à travers le monde (Marsh, 2017), avec une offre davantage présente en anglais, mais aussi considérable en français, notamment avec les plateformes FUN (http://fun-mooc.fr) et EDUlib (http://cours.edulib.org). Ils constituent un terreau fertile pour favoriser le développement professionnel des enseignants (Hodges et al, 2016; Seaton et al,

1. «Massive open online course », ou l'acronyme MOOC, est le terme le plus répandu pour parler des cours en ligne offerts gratuitement à de très larges publics. 
2015), en s'inscrivant en cohérence avec leurs besoins. Sur le site MOOC List (incluant les cours de Coursera, FutureLearn, Open2Study, etc.), on peut recenser plus de 250 MOOC anglophones avec le mot-clé " education », allant de la petite enfance (Early childhood education) aux méthodes pédagogiques (Learning mindsets et Skills) jusqu'aux cours plus réflexifs (What future for education?). Au Québec, on retrouve des MOOC en éducation sur l'innovation technopédagogique (Université de Montréal), sur le jeu ou la douance (Université du Québec à Trois-Rivières) ou sur la scénarisation pédagogique (OAS, Université de Montréal).

Par ailleurs, les MOOC connectivistes (cMOOC) permettent l'apprentissage actif des participants, qui sont appelés à créer des liens entre eux, à échanger des aspects de leur pratique et à favoriser le développement d'un réseau professionnel. Poellhuber et al. (2017) ont étudié le cas du MOOC ITES, qui s'adressait aux professionnels en enseignement supérieur soucieux de développer leur compétence en innovation pédagogique, comportait certaines composantes connectivistes et avait recours à l'apprentissage actif et à la collaboration. Le MOOC a regroupé plus de 1000 apprenants qui ont discuté et partagé à propos du numérique en éducation. Les entrevues menées auprès d'apprenants-clés, soit ceux qui ont participé activement aux activités du cours, ont su mettre en évidence le potentiel de développement professionnel de ce type d'activités, l'intention claire des participants de poursuivre les collaborations après le cours et leur volonté de parfaire leur compétence dans le domaine.

Pour le développement professionnel, le MOOC peut ainsi rejoindre les caractéristiques d'une stratégie efficace: une durée suffisante, des contenus ciblés et cohérents ainsi que des possibilités d'apprentissage actif et de collaboration. Toutefois, la grande variabilité, autant dans l'offre de formation que dans le degré d'implication des participants, aura une influence importante sur la transférabilité dans la pratique professionnelle.

\section{Les réseaux sociaux}

Les réseaux sociaux affichent le taux de pénétration le plus élevé dans les outils technologiques: plus d'un milliard d'utilisateurs en moins de dix ans pour Facebook (Radio-Canada, 2014). Sans contredit l'un des réseaux les plus répandus pour les usages personnels, Facebook offre un potentiel de partage professionnel évident, mais qui reste potentiellement trop en surface. Raby (2004) suggère qu'afin de s'approprier les TIC à des fins professionnelles, il faut d'abord en faire une appropriation pour des besoins personnels. Dans cette perspective, l'usage des groupes de discussion sur Facebook représente un moyen efficace pour rejoindre une communauté qui partage un intérêt commun, mais pour en faire une stratégie de développement professionnel efficace, il doit s'inscrire davantage dans le temps, avec des préoccupations liées aux réalités du terrain. Avec plus de 19000 membres, le groupe « Les TIC en éducation » constitue un exemple probant de réseautage. On peut également penser aux groupes plus spécifiques comme « Ressources à exploiter sur un TNI » (plus de 12000 membres); « La classe inversée - Partage et réussites » (plus de 4000 membres), «Profweb» (plus de 1000 membres), etc. Toutefois, force est de constater que dans leur grande majorité, les membres partagent et consultent des ressources uniquement. Bien que certains changements puissent s'amorcer par ce type d'activités, pour une réelle transformation de pratique, il doit y avoir un engagement ferme dans ce sens (Richard et al, 2017). De son côté, l'usage du réseau Twitter reste marginal dans la communauté enseignante, mais il devient rapidement un moyen de prédilection pour échanger sur les pratiques professionnelles de façon informelle chez ceux qui l'adoptent (Rehm et Notten, 2016). Le Sommet du numérique, organisé par le CRIFPE, permet de constater une certaine popularité envers l'outil : 8211 messages échangés en 2015, 6772 en 2016 et 3703 en 2017 
(données collectées par les auteurs). Malgré une décroissance annuelle illustrant un certain essoufflement, l'outil persiste auprès d'une communauté de technophiles. Parra et al. (2015) ont également fait l'analyse durant cinq années d'une conférence en sciences informatiques, entre 2009 et 2013. Ils ont plutôt constaté une croissance des messages, avec une réorientation des discussions vers des aspects plus informatifs et moins anecdotiques.

Le mot-clic \#TacEdChat (conversation du réseau TacTIC sur Twitter) a récolté près de 8000 messages dans les trois dernières années, alors que \#EduProf (discussion pédagogique mensuelle) a mené à 1835 messages entre le 12 avril 2020 et le 27 mai 2020. Les participants partagent sur Twitter des ressources, des nouvelles intéressantes, des points de vue, des vidéos, des astuces, etc., ce qui correspond également à ce que l'on observe sur Facebook. Ces utilisateurs sont des enseignants, des conseillers pédagogiques, des consultants, etc. Ils ont tous en commun une passion pour l'éducation et le numérique éducatif.

Finalement, les réseaux sociaux professionnels (Academia, ResearchGate et LinkedIn) sont des réseaux spécialisés contribuant aussi au développement professionnel d'intervenants et sont utilisés de façon plus fréquente en enseignement supérieur. Pour les chercheurs, ces plateformes permettent de diffuser les résultats de la recherche. Pour les enseignants, elles peuvent devenir un bon moyen de suivre les plus récents développements scientifiques spécifiques à leur domaine (Hobson et Cook, 2011) ou de favoriser les collaborations entre chercheurs (Al-Rahmi et al., 2015).

Toutefois, peu importe le réseau choisi, il existe un degré élevé de variabilité dans les usages. Pourquoi considérer les réseaux sociaux comme malléables? Peuvent-ils favoriser un développement professionnel efficace? Selon Macià et Garcia (2016), la réponse à ces questions n'est pas claire, car nous n'en savons pas assez sur les effets des réseaux sociaux sur le développement professionnel. En fait, les réseaux sociaux seront peu efficaces si leur utilisation consiste en de courtes visites non récurrentes, sans réel échange avec les pairs sur des besoins liés aux milieux. Au mieux, dans ce contexte, ils permettront d'amorcer une réflexion chez les utilisateurs ou encore de colliger de l'information (stratégie transmissive). $\mathrm{Si}$, au contraire, le réseau créé s'inscrit comme un complément à une stratégie plus complexe, avec une multitude d'échanges et de discussions, la communauté ainsi créée s'apparente plutôt à une stratégie à visée transformatrice pouvant correspondre, dans une certaine mesure, à des communautés d'apprentissage ou à des communautés professionnelles.

\section{Les stratégies à visée transformatrice}

Les stratégies dont la visée est transformatrice sont associées à la réflexion, à l'établissement de liens solides entre la théorie et la pratique, à la construction de connaissances et à leurs applications (Fraser et al., 2007). Selon ces mêmes auteurs, ces stratégies sont basées sur la collaboration et soutiennent l'autonomie sur les plans individuel et professionnel. Kennedy (2014) regroupe dans cette catégorie « tous les modèles et expériences qui incluent un élément lié à la résolution collaborative de problèmes et aux activités subséquentes, où ces dernières impliquent de s'interroger sur sa propre pratique et de mieux comprendre la pratique des autres, peut-être en s'engageant dans la recherche existante » [traduction libre] (p. 693). L'implication collective de plusieurs membres du personnel dans un processus de développement ou d'amélioration ainsi que différentes formes de projets liées à la recherche (recherche-action, design-based research ou recherche orientée par la conception) en sont des exemples. Un élément-clé souvent associé aux stratégies transformatrices est l'accès qu'ont les enseignants à des données issues des étudiants, nourrissant ainsi leur processus de développement 
professionnel. Il s'agit ici d'un processus pour alimenter la réflexion chez l'enseignant afin de l'amener à transformer sa pratique (Anderson, 2005).

Une démarche systématique de pratique réflexive peut s'inscrire dans un processus de scholarship of teaching and learning (SoTL), où l'enseignant s'intéresse particulièrement aux effets de son enseignement sur l'apprentissage des étudiants. Cette démarche -comme la recherche-action - permet à l'enseignant de réaliser un examen systématique de sa pratique d'enseignement en ayant comme «double objectif [...] d'améliorer l'enseignement et de contribuer à la connaissance théorique de manière à renforcer la qualité des apprentissages » (Colet et al, 2011, paragr. 7). L'enseignant adopte alors une posture qui se rapproche de celle du praticien chercheur (Bédard, 2014) et il pose ainsi son regard de praticien sur une problématique qu'il cerne et sur laquelle il fait de la recherche basée sur des résultats de ses observations, en collaboration avec un groupe.

Au collégial, on retrouve plusieurs initiatives de ce type. Cinq exemples représentent bien les stratégies transformatrices: le projet Cégep en réseau (Poellhuber et al., 2010), le projet SALTISE (Supporting Active Learning \& Technological Innovation in Studies of Education), le projet CLAAC (Classes d'apprentissage actif, voir Fournier St-Laurent et al., 2018), le projet Classe inversée (Poellhuber et al., 2020) et le projet d'innovation sur la persévérance et la réussite lié au PAN (Profweb, 2019). Ils ont tous en commun une longue durée, le recours à une combinaison de moyens diversifiés pour permettre le développement professionnel et des applications concrètes dans leur milieu respectif. Ces projets ont respectivement permis le développement des compétences sur la visioconférence Web, l'innovation pédagogique en sciences, les classes actives et les classes inversées. Le projet sur la classe inversée (Poellhuber et al., 2020), par exemple, a mis en œuvre un ensemble de moyens disséminés sur une période de trois ans. Des webinaires et des cours en ligne crédités ont été offerts aux enseignants, une communauté de pratique virtuelle regroupant des conseillers pédagogiques se réunissant aux trois semaines a été formée, un groupe de discussion sur Moodle a été constitué et des activités de visioconférence avec des experts ont été offertes en cohérence avec les besoins des participants. Une rencontre bisannuelle a favorisé le partage des rétroactions à partir des données de la recherche et la pratique réflexive sur les activités de classe a été rendue possible. Cette combinaison de moyens semble prometteuse pour soutenir le processus de développement professionnel des enseignants du postsecondaire, puisque cela s'inscrit sur une longue période (durée), avec une thématique précise ( «content focus»), tout en s'inscrivant dans une réalité de terrain (cohérence) de façon active et collaborative.

\section{Conclusion}

Comme nous l'avons évoqué au départ, les besoins de développement professionnel des enseignants au postsecondaire peuvent être grands, sur le plan tant pédagogique que disciplinaire. En plus d'être des spécialistes de leur discipline, les enseignants de ces niveaux doivent également être capables de transmettre leur savoir et de favoriser le développement des compétences de leurs étudiants.

Notre analyse des différents usages du numérique nous porte à croire que même si certains outils s'inscrivent davantage dans un type de stratégies, leur combinaison peut créer une réelle stratégie transformatrice permettant de développer les compétences. Une stratégie de développement professionnel alliant durée, adéquation avec le contenu universitaire, cohérence, apprentissage actif et participation collective peut ainsi être élaborée. Par exemple, un premier webinaire ou un MOOC au contenu pertinent et cohérent peut devenir la porte d'entrée vers un processus de 
développement professionnel transformateur en s'inscrivant dans une démarche plus large, faisant aussi appel à d'autres moyens tels qu'un partage via les réseaux sociaux, la participation à une communauté d'apprentissage en ligne ou en présentiel, ou encore l'accès à des données de la recherche.

Le numérique peut faciliter le développement professionnel, mais le développement professionnel n'est pas que numérique. L'expérience récente des MOOC témoigne du besoin des enseignants de poursuivre leur développement dans des situations authentiques, soit en créant des réseaux dans leur milieu, soit en participant à des évènements en présentiel (Bulger et al., 2015). L'offre des activités de développement (conférence, colloque, atelier) doit favoriser l'émergence de communautés de partage et d'échange qui s'étendront au-delà des limites de temps imposées lors de rassemblements. Ainsi, d'un côté, les formateurs doivent réfléchir à des approches mixtes (présentiel et à distance, synchrone et asynchrone) permettant de maximiser le potentiel de chaque activité, et de l'autre, les participants doivent s'ouvrir à intégrer à leur pratique de nouvelles façons de faire. Finalement, les milieux doivent mettre en place des facilitateurs (ressources physiques, espaces, temps, argent) pour permettre le développement professionnel des enseignants, notamment pour leur propre milieu, mais également en cohérence avec les besoins des autres milieux.

Le développement professionnel est un processus essentiel dans le contexte actuel de l'enseignement. Les enseignants sont des apprenants et ils apprennent en faisant, lisant et réfléchissant (comme les étudiants le font); en collaboration avec les autres enseignants; en regardant attentivement les étudiants et leurs travaux; et en partageant ce qu'ils voient (DarlingHammond et McLaughlin, 2011, p. 83). À la lumière de notre analyse, nous sommes convaincus que le numérique est un catalyseur et qu'il jouera dorénavant un rôle-clé dans une stratégie efficace de développement professionnel, composante essentielle de la dimension 9 de la compétence numérique.

\section{Références}

Al-rahmi, W. M., Othman, M. S. et Yusuf, L. M. (2015). The effect of social media on researchers' academic performance through collaborative learning in Malaysian higher education. Mediterranean Journal of Social Sciences, 6(4), 193-203. https://doi.org/10.5901/mjss.2015.v6n4s1p193

Anderson, T. (2005). Design-based research and its application to a call centre innovation in distance education. La revue canadienne de l'apprentissage et de la technologie, 31(2). http://learntechlib.org/p/42762

Bédard, D. (2014). Être enseignant ou devenir enseignant dans le supérieur : telle est la question... de posture! Dans G. Lameul et C. Loisy (dir.), La pédagogie universitaire à l'heure du numérique (p. 97-110). De Boeck.

Beichner, R. J., Saul, J. M., Abbott, D. S., Morse, J. J., Deardorff, D., Allain, R. J., Bonham, S. W., Dancy, M. H. et Risley, J. S. (2007). The student-centered activities for large enrollment undergraduate programs (SCALE-UP) project. Dans E. F. Redish et P. J. Cooney (dir.), Reviews in physics education research: Vol. 1 (p. 2-39). PER-Central. http://compadre.org/per/...

Bolduc, B. (2010). Un aperçu du champ d'exercice de la profession enseignante au collégial. Bulletin Formation et profession, 17(1), 9-14. http://crifpe.ca/download/verify/838 
Bulger, M., Bright, J. et Cobo, C. (2015). The real component of virtual learning: Motivations for face-to-face MOOC meetings in developing and industrialised countries. Information, Communication \& Society, 18(10), 1200-1216. https://doi.org/10.1080/1369118X.2015.1061571

Colet, N. R., McAlpine, L., Fanghanel, J. et Weston, C. (2011). Le concept de scholarship of teaching and learning : la recherche sur l'enseignement supérieur et la formalisation des pratiques enseignantes. Recherche et formation, (67), 91-104.

https://doi.org/10.4000/rechercheformation.1412

Conseil supérieur de l'éducation. (2014). Le développement professionnel, un enrichissement pour toute la profession enseignante. Gouvernement du Québec.

https://www.cse.gouv.qc.ca/...

Darling-Hammond, L. et McLaughlin, M. W. (2011). Policies that support professional development in an era of reform. Phi Delta Kappan, 92(6), 81-92. https://doi.org/10.1177/003172171109200622

Deschênes, M. (2014). Le web social, un levier de développement professionnel? [rapport de recherche]. Collège O'Sullivan de Québec. http://iteractive.ca/devpro

Desimone, L. M. (2009). Improving impact studies of teachers' professional development: Toward better conceptualizations and measures. Educational Researcher, 38(3), 181-199. https://doi.org/10.3102/0013189X08331140

Fournier St-Laurent, S., Normand, L., Bernard, S. et Desrosiers, C. (2018). Les conditions d'efficacité des classes d'apprentissage actif. Rapport de recherche PAREA. Collège Ahuntsic. https://eduq.info/xmlui/handle/11515/35536

Fraser, C., Kennedy, A., Reid, L. et Mckinney, S. (2007). Teachers' continuing professional development: Contested concepts, understandings and models. Journal of In-Service Education, 33(2), 153-169. https://doi.org/10.1080/13674580701292913

Freeman, S., Eddy, S. L., McDonough, M., Smith, M. K., Okoroafor, N., Jordt, H. et Wenderoth, M. P. (2014). Active learning increases student performance in science, engineering, and mathematics. Proceedings of the National Academy of Sciences, 111(23), 8410-8415. https://doi.org/10.1073/pnas.1319030111

Garet, M. S., Porter, A. C., Desimone, L., Birman, B. F. et Yoon, K. S. (2001). What makes professional development effective? Results from a national sample of teachers. American Educational Research Journal, 38(4), 915-945. https://doi.org/10.3102/00028312038004915

Gast, I., Schildkamp, K. et van der Veen, J. T. (2017). Team-based professional development interventions in higher education: A systematic review. Review of Educational Research, 87(4), 736-767. https://doi.org/10.3102/0034654317704306

Guskey, T. R. (2000). Evaluating professional development. Corwin.

Hamel, C., Laferrière, T., Turcotte, S. et Allaire, S. (2013). Un regard rétrospectif sur le développement professionnel des enseignants dans le modèle de l'École éloignée en réseau. Sticef, 20, 105-130. http://sticef.univ-lemans.fr/... 
Hobson, J. et Cook, S. (2011). Social media for researchers: Opportunities and challenges. MAI Review, (3). http://review.mai.ac.nz/...

Hodges, C., Lowenthal, P. et Grant, M. (2016). Teacher professional development in the digital age: Design considerations for MOOCs for teachers. Dans Proceedings of Society for Information Technology \& Teacher Education International Conference (p. 2075-2081). Association for the Advancement of Computing in Education.

Karsenti, T. et Grégoire, P. (2015). Professionnalisation et développement professionnel des enseignants dans un contexte d'intégration des TICE : le cas du Québec. Distances et Médiations des Savoirs, (11), 1-9. https://doi.org/10.4000/dms.1140

Kennedy, A. (2005). Models of continuing professional development: A framework for analysis. Journal of In-Service Education, 31(2), 235-250. https://doi.org/10.1080/13674580500200277

Kennedy, A. (2014). Understanding continuing professional development: The need for theory to impact on policy and practice. Professional Development in Education, 40(5), 688-697. https://doi.org/10.1080/19415257.2014.955122

Macià, M. et García, I. (2016). Informal online communities and networks as a source of teacher professional development: A review. Teaching and Teacher Education, 55, 291-307. https://doi.org/10.1016/j.tate.2016.01.021

Marsh, N. (2017, 4 janvier). MOOC users reach 58 million globally. The Pie News. http://thepienews.com/...

Martinet, M. A., Raymond, D. et Gauthier, C. (2001). La formation à l'enseignement : les orientations, les compétences professionnelles. Gouvernement du Québec, ministère de l'Éducation. http://education.gouv.qc.ca/...

Ministère de l'Éducation et de l'Enseignement supérieur. (2019). Cadre de référence de la compétence numérique. Gouvernement du Québec. http://education.gouv.qc.ca/...

Olmstead, A. et Turpen, C. (2016). Assessing the interactivity and prescriptiveness of faculty professional development workshops: The real-time professional development observation tool. Physical Review Physics Education Research, 12(2), article 020136. https://doi.org/10.1103/PhysRevPhysEducRes.12.020136

Parra, D., Trattner, C., Gómez, D., Hurtado, M., Wen, X. et Lin, Y. R. (2016). Twitter in academic events: A study of temporal usage, communication, sentimental and topical patterns in 16 computer science conferences. Computer Communications, 73, 301-314. https://doi.org/10.1016/j.comcom.2015.07.001

Patton, K., Parker, M. et Pratt, E. (2013). Meaningful learning in professional development: Teaching without telling. Journal of Teaching in Physical Education, 32(4), 441-459. https://doi.org/10.1123/jtpe.32.4.441

Penuel, W. R., Fishman, B. J., Yamaguchi, R. et Gallagher, L. P. (2007). What makes professional development effective? Strategies that foster curriculum implementation. American Educational Research Journal, 44(4), 921-958. https://doi.org/10.3102/0002831207308221 
Poellhuber, B., Allen, C. et Roy, N. (2010). Projet Cégeps en réseau. Rapport final - Volet technopédagogique de la recherche. CEFRIO. http://cefrio.qc.ca/...

Poellhuber, B., Roy, N., Caron, F., Chouinard, R., Meyer, F., Lison, C. et Laberge, V. (2020). La classe inversée : une recherche-action-formation pour développer une approche ayant un impact sur l'engagement, la motivation et la réussite [rapport de recherche]. Fonds de recherche du Québec - Société et culture. http://frqsc.gouv.qc.ca/...

Poellhuber, B., Roy, N. et Moukhachen, M. (2017). Les stratégies d'apprentissage et de collaboration déployées par des utilisateurs-clés dans le MOOC « Innovations technopédagogiques en enseignement supérieur (ITES) ». Raisons éducatives, 2017/1(21), 209-228. https://doi.org/10.3917/raised.021.0209

Prince, M. (2004). Does active learning work? A review of the research. Journal of Engineering Education, 93(3), 223-231. https://doi.org/10.1002/j.2168-9830.2004.tb00809.x

Profweb (2019, 6 décembre). Expérimentation de la classe inversée bonifiée par les technologies : un projet panquébécois du Plan d'action numérique. http://profweb.ca/...

Raby, C. (2004). Analyse du cheminement qui a mené des enseignants du primaire à développer une utilisation exemplaire des technologies de l'information et de la communication en classe [thèse de doctorat, Université du Québec à Montréal, Canada]. Archive TeL. https://tel.archives-ouvertes.fr/edutice-00000750

Raby, C., Karsenti, T., Meunier, H. et Villeneuve, S. (2011). Usage des TIC en pédagogie universitaire : point de vue des étudiants. Revue internationale des technologies en pédagogie universitaire, 8 (3), 6-19. https://doi.org/10.7202/1006396ar

Radio-Canada (2014, 4 février). Facebook a 10 ans et 1,2 milliard d'amis. http://ici.radiocanada.ca/...

Rehm, M. et Notten, A. (2016). Twitter as an informal learning space for teachers!? The role of social capital in Twitter conversations among teachers. Teaching and Teacher Education, 60, 215-223. https://doi.org/10.1016/j.tate.2016.08.015

Réseau REPTIC (s.d.-a). Le Réseau. Récupéré le 2 juillet 2020 de http://reptic.qc.ca/le-reseau

Réseau REPTIC (s.d.-b). Pratiques technopédagogiques novatrices. Repéré le 11 août 2020 à http://reptic.qc.ca/...

Richard, M., Carignan, I., Gauthier, C. et Bissonnette, S. (2017). Quels sont les modèles de formation continue les plus efficaces pour l'enseignement de la lecture et de l'écriture chez les élèves du préscolaire, du primaire et du secondaire? Une synthèse des connaissances [rapport de recherche]. Fonds de recherche du Québec - Société et culture. https://r-libre.teluq.ca/1099

Seaton, D. T., Coleman, C. A., Daries, J. P. et Chuang, I. (2014, 27 octobre). Teacher enrollment in MITx MOOCs: Are we educating educators? (MITx Working Papers, $\mathrm{n}^{\circ} 13$ ). MIT Office of Digital Learning et HarvardX Research Committee. http://hdl.handle.net/1721.1/96661

Stes, A., Min-Leliveld, M., Gijbels, D. et Van Petegem, P. (2010). The impact of instructional development in higher education: The state-of-the-art of the research. Educational Research Review, 5(1), 25-49. https://doi.org/10.1016/j.edurev.2009.07.001 
St-Pierre, L. et Lison, C. (2009). Une formation continue à mon image. Étude de caractéristiques des enseignantes et des enseignants des collèges francophones membres de Performa en relation avec la formation continue [rapport de recherche]. Université de Sherbrooke / Secteur Performa. http://usherbrooke.ca/performa/...

Uwamariya, A. et Mukamurera, J. (2005). Le concept de « développement professionnel » en enseignement : approches théoriques. Revue des sciences de l'éducation, 31(1), 133-155. https://doi.org/10.7202/012361ar

Wei, R. C., Darling-Hammond, L., Andree, A., Richardson, N. et Orphanos, S. (2009). Professional learning in the learning profession: A status report on teacher development in the United States and abroad [rapport technique]. National Staff Development Council. https://learningforward.org/... 